\title{
An Introduction to Quantum Computers
}

\author{
Christof Zalka* \\ zalka@t6-serv.lanl.gov
}

February 1, 2008

\begin{abstract}
This is a short introduction to quantum computers, quantum algorithms and quantum error correcting codes. Familiarity with the principles of quantum theory is assumed. Emphasis is put on a concise presentation of the principles avoiding lengthy discussions.
\end{abstract}

\section{Quantum Computers}

A quantum computer is a collection of 2-level systems (qubits). Thus the quantum computer $(\mathrm{QC})$ is described by a vector in a Hilbert space which is the tensor product of 2-dimensional Hilbert spaces. With $l$ qubits this space has dimension $2^{l}$.

The state of an l-qubit quantum register can be written as a superposition of the "computational basis states". These are the states where each qubit is in one of its two basis states $|0\rangle$ or $|1\rangle$. We label these basis states by the integer which they represent in binary. Thus:

$$
\mid \text { register }\rangle=\sum_{n=0}^{2^{l}-1} c_{n}|n\rangle
$$

To compute, a quantum computer makes a sequence of unitary transformations. Each unitary transformation acts on a small set of qubits by using "exterior fields" which can effectively be treated classically. Thus if, e.g., a qubit is realized by the 2-level approximation of an atom, we can induce some $\mathrm{U}(2)$ transformation by applying an electromagnetic field with the right frequency for a certain time and with a specific phase. If no such field is applied we assume that the state of the QC doesn't change.

A special case of unitary transformations are permutations of the basis states; e.g., a NOT which flips the $|0\rangle$ and $|1\rangle$ states of a qubit or a so-called controlled-NOT acting as follows on the basis states of 2 qubits:

*Supported by Schweizerischer Nationalfonds and LANL 


$$
U_{\mathrm{CNOT}}|a, b\rangle=|a, a \mathrm{XOR} b\rangle \quad a, b=0,1
$$

Together with the controlled-controlled-NOT (CCNOT or Toffoli gate)

$$
\text { CCNOT }: \quad|a, b, c\rangle \rightarrow \mid a, b,(a \text { AND } b) \text { XOR } c\rangle,
$$

we get a so-called "universal set" of gates for what is called "reversible computation". In reversible computation every gate has as many output bits as input bits and because the gate has to be reversible (1 to 1 ), only permutations of the possible input states are allowed. It is not difficult to see that with the above 3 gates we can compute any function of a binary input, just as we can with conventional computation. (The CCNOT can be used as AND.)

When starting with a superposition of all "computational" basis states, such as (1) the quantum computer can compute a superposition of outputs of a function for all possible $2^{l}$ inputs. Now, at the end of the quantum computation we have to make a measurement on the quantum computer. We measure for each qubit whether it is in state $|0\rangle$ or $|1\rangle$. Thereby we collapse the state of the QC onto a computational basis state $|n\rangle$ with probability $\left|c_{n}\right|^{2}$.

If we do this to a superposition of function values, this is not going to be of interest. The trick is to look for interference between the computational basis states. For this we have to add "non-classical" gates to the above ones, that is, gates which transform computational basis states into a superposition thereof.

Shor's quantum algorithm for factoring large integers [1] first computes a superposition of functional values and then applies a number of "non-classical" gates before measuring the QC. The non-classical gates bring the $\mathrm{QC}$ into a state where only about square root of the computational basis states have a sizable amplitude (coefficient $c_{n}$ ), thus the final measurement will pick one of them. The observed basis state $|n\rangle$ will thus have a "random component" but also carries some information which can be used to solve the mathematical problem at hand. Shor's algorithm is described in more detail in section 3.1.

\section{Possible technical realizations: ions in an elec- tromagnetic trap}

One proposal [2] for building a quantum computer is to use a linear ion trap. In such a trap a number of ions will line up along the $z$-axis. Along the $x$ and $y$-axis they are strongly confined by a high-frequency electric field that switches between being confining in the $x$-direction and deconfining in the $y$ direction and vice versa. The net effect is that the ions are confined strongly in both directions. In the $z$-direction the ions are confined by a relatively weak harmonic electric potential. Due to their mutual electrostatic repulsion the ions will form a string with separations of the order of micro meters. 
Each ion represents a qubit. By shining laser light at an individual ion, $\mathrm{U}(2)$ transformations can be applied to that qubit. For "universal quantum computation" we at least also need to be able to induce unitary transformations on a pair of ions such that the initial product states will become non-product states ("entangled" states).

To do this, the ions have to be cooled so that they occupy the lowest energy state of their motional degrees of freedom in the confining potential. It is not so difficult to do this for the strongly confining $x$ - and $y$-directions. For the $z$-direction, instead of looking at the individual motions of the ions, one looks at collective motions, "normal modes" which are like uncoupled harmonic oscillators. The lowest such mode is the "center-of-mass" mode where the ions can be imagined to oscillate synchronously without changing their spacings.

The idea now is to use the two lowest states of this center-of-mass oscillator as a "bus-qubit". The internal state of an ion can be coupled to the "center-ofmass" degree of freedom, e.g., by shining laser light at it with a frequency which will take the ground state of the ion and the center-of-mass motion to the first excited state of both.

This has been done experimentally with a single ion. Also pictures of some 30 ions aligned in a linear ion trap (and fluorescing in laser light) have been obtained, but presently the main problem is to cool such a string of ions to the motional ground state. One tries to achieve this with laser-cooling (dopplercooling and others). See e.g. [3].

\section{Quantum algorithms}

\section{1 factoring large integers}

The quantum factoring algorithm arguably is the only known case where a quantum computer could solve an interesting problem much faster than a conventional computer. Actually the computation time is a small power $\left(2^{\text {nd }}\right.$ or $\left.3^{\text {rd }}\right)$ of the number of digits of the integer to be factored. The fastest known classical integer-factoring algorithms use super-polynomial time, and it is believed that no polynomial-time such algorithms exist.

Shor's quantum factoring algorithm relies on the fact that factoring can be "reduced" to the problem of finding the period of the following periodic function:

$$
f(x)=a^{x} \bmod N \quad,
$$

where $N=p \cdot q$ is the number to be factored and $a$ is essentially an arbitrary constant. Note that Euler's generalization of Fermat's little theorem states that $a^{(p-1)(q-1)} \bmod (p q)=1$. Thus $(p-1)(q-1)$ is a multiple of the period of $f(x)$. It should therefore be plausible that there are efficient ways to get the factors $p$ and $q$ from the period. 
After computing a superposition of the form $\sum|x, f(x)\rangle$, the period can be found by employing the "quantum Fourier transform" which applies the discrete Fourier transform to the $2^{l}$ amplitudes of a quantum register.

To obtain $|x, f(x)\rangle$ with reversible computation we have to make a little detour. By using additional qubits in the state 0 , we can use CNOT and CCNOT to compute XOR and AND, but we also produce unwanted output bits. In a superposition, the unwanted qubits will be quantum-correlated (entangled) with the wanted qubits. Observing and resetting the unwanted qubits doesn't work, as we thereby also collapse the wanted part of the QC. The trick is to first compute $f(x)$ including the garbage $g(x)$, then copy $f(x)$ into a "save" register and then undo the first step, which is of course possible in reversible computation:

$$
|x, 0,0,0\rangle \rightarrow|x, f(x), g(x), 0\rangle \rightarrow|x, f(x), g(x), f(x)\rangle \rightarrow|x, 0,0, f(x)\rangle
$$

The copying of $f(x)$ in the second step can be done with a sequence of CNOT's.

So by starting with a superposition with equal amplitudes of all $x$, we get:

$$
\frac{1}{\sqrt{2^{l}}} \sum_{n=0}^{2^{l}-1}|x, 0\rangle \quad \rightarrow \quad \frac{1}{\sqrt{2^{l}}} \sum\left|x, a^{x} \bmod N\right\rangle
$$

For the following it is easier to imagine that now we measure the second register, but it is not necessary. After such a measurement the first register will be in a superposition of all $x$ that give the measured output value. The value of the smallest such $x$ is random, but the spacing of the following values is the period of $f(x)$ which we want to know. Thus the amplitudes in the first register are peaked with constant spacings between the peaks, but everything is shifted by a random value. When applying the quantum Fourier transform to the first register we will again get amplitudes that are peaked at regular intervals, but now the first peak is at the origin and the random shift in the previous peaks only shows up as some complex phase of the peaks. (To get sharp peaks we choose the size of the $x$-register such that $2^{l}$ is at least of the order of the period squared.) By measuring the Fourier transformed register, and repeating the whole quantum computation a few times, one obtains the spacing of the peaks.

The quantum Fourier transform is done by using the fast Fourier transform algorithm (FFT), which applies very naturally to transforming the amplitudes of a quantum register. Actually it can be done so efficiently that it is negligible for the overall computation time.

\section{2 unstructured search}

To search through $N$ cases, there is a simple quantum algorithm [4 that takes some $\sqrt{N}$ steps. This is not a very strong improvement over the classical case 
with $N$ steps. Furthermore it can be shown that for this problem no better quantum algorithm exists [5, 6. To prove this, the unstructured search is formalized with a so-called "oracle", a black-box subroutine which gives output 1 only for one out of all possible inputs.

\section{3 simulating arbitrary quantum systems}

The amplitudes of the computational basis states of a QC can be made to follow the time evolution of the amplitudes of essentially any quantum system [7, 8]. Obtaining information about the quantum state is then of course restricted by the same fundamental quantum principles as it is for the original quantum system.

Say we have a quantum mechanical Hamilton operator that is a sum of a kinetic and a potential term, thus a sum of a term which is a function of momentum operators and a term that is a function of position operators. We now discretize the wave function of this quantum mechanical system and "store" it as the amplitudes of the computational basis states of the QC. The point is that we can go to momentum space by applying the quantum Fourier transform. Then we will have the discretized wavefunction in momentum space. Time evolution is implemented by evolving the wavefunction for a short time only according to the potential term, then go to momentum space and evolve according to the kinetic term, and so on.

Evolving the wavefunction for a short time according to e.g. only the potential term, amounts to multiplying with a complex phase. We have to carry out a transformation of the form $|x\rangle \rightarrow e^{i f(x)}|x\rangle$, which can be done quite easily.

\section{Quantum error correcting codes}

To carry out a long quantum computation seems to require very precise operations and low noise. From imprecisions in the applications of the exterior fields (lasers, etc.) quantum gates will always be somewhat different from the intended unitary operations. In this respect we have the same problems as with an analog computer where, contrary to digital hardware, slightly deviating values are not automatically reset to a standard value. Also it is very difficult to isolate the degrees of freedom of a quantum computer from the environment.

Therefore quantum computation might well be practically impossible, were there not the possibility of quantum error correction. Interestingly, it is possible to correct (continuous) quantum amplitudes much better than e.g. continuous classical quantities in an analog computer.

To simplify things, the following discussion will not encompass the most general possibilities for quantum error correction. I will describe how the 5-

qubit error correcting code [10] works, which has been shown to be the shortest possible quantum code. 
So we want to encode (and thus protect) a qubit in 5 qubits. The code is a 2 dimensional subspace of the 32 dimensional Hilbert space of the code. How can we correct for errors? Making a full (no degenerate eigenvalues) measurement of the code in order to correct for errors is not good as this will collapse the (generally unknown) encoded qubit. The trick is to only measure what error has affected the code without learning anything about the encoded qubit. The eigenspaces of the error measurement have to be at least 2 dimensional so that the encoded quantum information will not collapse.

An error correcting code can of course only correct for some errors, ideally the most probable ones. The standard assumption is that the probabilities of errors affecting different physical qubits are independent. Then it makes sense to correct for all errors which affect just one qubit.

For every qubit there are 3 such errors, namely bit flips, phase flips and bit-phase flips, corresponding to the 3 Pauli matrices. We must also take into account that no error may have happened. Then for the 5 -qubit code we have $5 \cdot 3+1$ possible "errors". After having been exposed to noise the code will in general be in a superposition of the 16 resulting states, but a measurement of the error will collapse it to one of these states. Thus for convenience we can simply imagine that one of the 16 errors has happened.

Now let's see what conditions the code (= the 2-dim. code subspace) has to fulfill. The condition is that the 16 images under the error operators of the 2-dim. code subspace have to be pairwise orthogonal. This makes it possible to construct an "error observable" with these eigenspaces. Note that the 16 mutually orthogonal 2-dim. subspaces "fill" the 32 dimensional code Hilbert space, which is why the 5 qubit code is called "perfect".

Once we have determined which error has happened we simply undo this error (it's a unitary transformation). It may technically not be feasible to directly measure the error observable, but one can apply a series of quantum gates such that the error observable then corresponds to simply measuring 4 of the 5 qubits.

Such codes can, e.g., be used to store an (unknown) quantum state or transmit it through a noisy transmission line. In quantum computing we also must be concerned about the noisiness of the error correction operations. Also, we never want to decode the processed quantum information, as this would expose it to noise. Schemes have been developed for "fault tolerant quantum computing" where error correction operations can increase the probability of having an undisturbed state even though they are noisy themselves, and operations (quantum gates) can be applied to encoded qubits such that again some level of noise can be tolerated. If we imagine one of our standard errors $E_{i}$ to happen suddenly with some probability, then such schemes can tolerate a certain number of such errors before the quantum computation goes wrong, provided that the errors don't cluster too much. Without fault tolerant quantum computing, a single error would already be too much.

One can think of iterating the encoding procedure, thus, e.g., one qubit 
would be encoded in five qubits which in turn would each be encoded in five qubits. It has been shown that with such a scheme arbitrarily long quantum computations could be carried out once the rate of errors per physical qubit is below a certain level of the order of $10^{-4}$ per operation (see e.g. 14]).

\section{$5 \quad$ Recommended papers}

\section{introductions:}

15 is a semi-popular short introduction.

Also John Preskill gives a lecture on quantum computation at Caltech. The Web site [16] contains the lecture notes and other useful information and links.

\section{factoring:}

Shor's paper [1] is well written but long. A short account of Shor's algorithm is also given in 13.

\section{error correction:}

In [9] Shor describes how his 9-qubit quantum error correcting code works. This was the first quantum code.

\section{fault tolerant quantum computing:}

In 12. Shor describes how to carry out fault tolerant error correction and fault tolerant operations on the 7 -qubit and related codes.

\section{References}

[1] Peter W. Shor (AT\&T Research), Polynomial-Time Algorithms for Prime Factorization and Discrete Logarithms on a Quantum Computer, SIAM J. Computing 26, 1484 (1997), also quant-ph/9508027

[2] J.I. Cirac and P. Zoller, Quantum Computations with Cold Trapped Ions, Phys. Rev. Lett. 74, No. 200, 4091 (May 1995)

[3] Ch. Monroe and J. Bollinger, Atomic physics in ion traps, Physics World, March 1997

[4] L. Grover, A fast quantum mechanical algorithm for database search, Proceedings, 28th Annual ACM Symposium on the Theory of Computing (STOC), May 1996, pages 212-219, also quant-ph/9605043

[5] C. Bennett et al., Strength and Weaknesses of Quantum Computing, SIAM Journal on Computing, 26, No. 5, 1510-1524 (Oct. 1997), also quant$\mathrm{ph} / 9701001$ 
[6] Ch. Zalka, Grover's quantum searching algorithm is optimal, quant$\mathrm{ph} / 9711070$

[7] Ch. Zalka, Simulating quantum systems on a quantum computer, Proceedings of the Royal Society, 8 January 1998 Series A, 454, No. 1969, 313-322, also quant-ph/9603026

[8] Stephen Wiesner, Simulations of Many-Body Quantum Systems by a Quantum Computer, quant-ph/9603028

[9] P. Shor, Scheme for reducing decoherence in quantum computer memory Phys. Rev. A 52, No. 4, pp. R2493-R2496 (1995)

[10] R. Laflamme, C. Miquel, J.P. Paz, W. Zurek, Perfect quantum error correcting code, Phys. Rev. Lett. 77, No. 1, 198-201 (1996), also quant-ph/9602019

[11] A.R. Calderbank and P. Shor, Good Quantum Error-Correcting Codes Exist, Phys. Rev. A 54, No. 2, 1098-1106 (1996), also quant-ph/9512032

[12] P. Shor, Fault-tolerant quantum computation, 37th Symposium on Foundations of Computing, IEEE Computer Society Press, 1996, pp. 56-65, also quant-ph/9605011

[13] I. Chuang, R. Laflamme, P. Shor, W. Zurek, Quantum Computers, Factoring, and Decoherence, Science 270, 1633-1635 (1995), also quant$\mathrm{ph} / 9503007$

[14] Ch. Zalka, Threshold estimate for Fault Tolerant Quantum Computation, quant-ph/9612028

[15] B. Cipra, Quantum Computation, SIAM news 31, No. 8 (Oct. 98)

[16] John Preskill, Lecture notes on Quantum Computation, obtainable at http://www.theory.caltech.edu/people/preskill/ph229/ 